\title{
Improved Scanning Methods for Wavelet Coefficients of Video Signals
}

Fabrice Rossetti, Jiro Katto and Mutsumi Ohta Information Technology Research Laboratories, NEC Corporation.

1-1, Miyazaki 4-chome, Miyamae-ku

Kawasaki-shi, KANAGAWA 216, JAPAN

E-mail: katto@dsp.cL.nec.co.jp 
The number of pages: 9

The number of tables: 1

The number of figures: 15

Running headline: Improved Scanning Methods 


\section{Introduction}

A wavelet transform has been a promising method for image and video compression. It can effectively reduce blocking artifacts and mosquito noises in addition to its good energy compaction properties, thus leading to improvement of subjective quality $[1,2]$. Introduction of this wavelet transform to the image coding fields was firstly discussed by Mallat [3,4] and Daubechies [5]. Since then, it becomes very popular and has been introduced to still image compression [6] and video compression $[1,2,7,8]$. It can be mathematically related to the subband coding $[9,10]$, Laplacian pyramid coding [11] and the transform coding as reported in some literatures $[5,12,13,14]$. This means that developed strategies for each coding technique can be introduced into the wavelet transform straightforwardly. A point lies in their skillful modification utilizing properties of the wavelet transform.

There are a lot of works which treat the wavelet transform as the subband coding. In this case, coding process is applied to each subband created by the wavelet transform. On the other hand, Ohta et al. proposed a transform coding (block based) approach in which wavelet transformed coefficients lying

in a spatially neighboring position are gathered and encoded in a block-by-block manner $[1,2]$. This approach is appropriate for implementation because only a limited amount of coefficients are needed at a given time and pipeline calculation can be introduced. Scanning of the wavelet transformed coefficients, however, has not been investigated sufficiently so far, including a comparison between the subband based approach and the block based approach. Therefore, scanning methods to improve coding efficiency of video coding algorithms using the wavelet transform are studied in this paper.

Section 2 states the analysis of statistical properties of the wavelet transformed image sequence, which is the basis of this paper. In Section 3, different scanning patterns are presented for the block based approach based on the statistical analyses of Section 2. Scanning algorithms to reduce bit rates are also considered; simple scanning, zonal scanning and conditional scanning. In Section 4, scanning algorithms for the subband based approach are proposed, and the effect of a block-wise non-linear filter removing isolated non-zero coefficients in high frequencies is investigated. Section 5 presents simulation results, and Section 6 concludes this paper.

\section{Statistical Properties of the Wavelet Transform}

\subsection{A Brief Review of the Wavelet Transform}

The wavelet transform can be represented by a filterbank as shown in Figure 1. It has an interesting property; the image resolution becomes high for high frequency components and low for lower frequency components. This property emulates human visual system [3, 4], and is also useful to reduce mosquito noises $[1,2]$. Two-dimensional frequency division by the wavelet transform is shown in Figure 2 . This 
is very similar to the Laplacian pyramid coding [11], and is effective in data compression of highly correlated inputs such as image signals.

There are two ways to introduce the wavelet transform to video coding. The first one is applying it to input frames before taking frame difference, and the second one is applying it to frame difference signals after motion compensation [15]. In this paper, the latter approach is chosen similar to our previous works $[1,2,8]$. Overlapped motion compensation efficiently reduce prediction errors, and good coding efficiency has been already achieved.

The coefficients of the wavelet transform can be collected in two different ways, taking either all coefficients belonging to one frequency or by grouping points from the spatially same location together. The former is referred as subband coding, while the latter is similar to transform coding. In either case, data compression properties can be improved by applying efficient entropy coding.

In the following of this section, statistical properties of the wavelet transform to frame difference signals using real CIF [16] image sequences are investigated. The four tap orthogonal filters [5] are used, and the band splitting pattern is fixed as depicted in Figure 2. Resultant properties are utilized for designing efficient scanning methods described later.

\subsection{Energy Distribution}

Analogous to $[17,18]$ for DCT, the mean energy of the frame difference signals after wavelet transform is calculated. If good energy compaction is achieved, fewer coefficients must be coded and bits amount will be saved. Figure 3 shows results for four CIF image sequences. $V_{3}$ has the most energy, followed by $W_{3}^{L H}$. It is also shown that the energy content is higher in the $W_{n}^{L H}$ subband than in the $W_{n}^{H L}$ subband. Furthermore, the energy of $W_{n}^{L H}$ is higher than $W_{n+1}^{H H}$ in general. These results give us a guideline when deciding in which order subbands should be scanned.

\subsection{Correlation}

Correlation tells us how far redundancy can be removed. For our purposes, calculating the correlation of adjacent coefficients is sufficient. The values of interframe prediction errors are shown in Figure 4. Correlation is resolution independent, i. e. the values do not vary significantly from one resolution layer to another. We can see that the horizontal correlation is strongest in $V_{3}$. In $W_{3}^{H L}$, the vertical direction predominates slightly and in $W_{3}^{L H}$, it is again the horizontal direction dominating.

Another problem is conditional probabilities between the different resolution layer of the same filtering direction. One can think of the subband picture $W_{1}^{H L}$ as a zoom-in of the subband picture $W_{2}^{H L}$. A zoom-in of a picture should resemble pretty much to the more coarse picture. Therefore, the lower resolution picture and the higher resolution picture are expected to be correlated. 
Figure 5 shows the link between different layers from the viewpoint of conditional probabilities. The coefficients linked by the line should be correlated as they represent the same part of the picture but at different frequencies. There are two interesting quantities: the probability of having a non-zero (NZ) value in the lower frequency layer for a zero $(\mathrm{Z})$ value in the higher one, and the probability of having a non-zero value in the lower frequency layer for a non-zero value in the higher one. These quantities are depicted in Figure 6, where $T$ is a threshold value for quantization and $L$ is the number of layers. The fact that the first probability is very small means that, when the lower frequency component is zero, the corresponding higher one also tends to be zero. The second probability suggests that there exist higher chances of finding a non-zero value in higher frequency when the corresponding lower one is also non-zero. These tendencies hardly depend on resolutions.

\subsection{Discussions}

Results of the statistical analyses suggest following properties. Figure 3 shows that

1. $V_{3}$ has the most energy followed by $W_{3}, W_{2}$ and $W_{1}$.

2. Within one layer, $W_{n}^{L H}$ has the highest energy, followed by $W_{n}^{H L}$ and $W_{n}^{H H}$.

3. Between neighboring layers, $W_{n}^{L H}$ often has slightly higher energy than $W_{n+1}^{H H}$.

Figure 4 suggests that

4. $W_{n}^{L H}$ and $W_{n}^{H H}$ have their strongest correlation in horizontal direction, and $W_{n}^{H L}$ does in vertical direction.

Finally, Figure 6 indicates that

5. There exists a correlation between coefficients in one layer to another layer in the same filtering direction i. e. between coefficients in $W_{n}^{x y}$ and $W_{n+1}^{x y}$.

\section{Block Based Approach}

\subsection{Scanning Patterns}

A scanning pattern defines the order of reading and sending the transform coefficients. The basic idea is to start scanning the values which have a high probability of being non zero and scanning the values with a high probability of being zero at the end. Similar to the zigzag scanning for DCT [18], various scanning patterns for the wavelet transform are designed, keeping the properties stated in the previous section in mind. 
One block is composed of $8 \times 8$ transform coefficients which are located in a spatially neighboring position. For each block, four different scanning patterns are defined as depicted in Figure 7:

Pattern 1 This pattern is determined by taking account of the power distribution of subbands and the directional correlation in each subband. (the properties 1, 2 and 4 are used).

Pattern 2 The order of scanning of subbands is slightly changed from the pattern 1 . The motivation is the property 3 .

Pattern 3 Different from the pattern 1, successively scanned points between subbands are determined according to their spatial position. This is the expanded idea from the property 5 .

Pattern 4 This pattern is just an analogy of the peano scan, which is known as the spatially densest curve exploiting isotropic spatial correlation if it exist.

Our objective lies in finding the best pattern among these boustrophedon scanning methods. Performance comparison will be carried out in Section 5 .

\section{$3.2 \quad$ Scanning Algorithms}

Scanning for itself doesn't make a lot of sense unless a variable length coding scheme is introduced. Similar to the conventional DCT based approach, introduction of a two-dimensional variable length coding is assumed. One axis of the VLC table consists of the magnitude of a non zero value and the other axis consists of the number of zeros preceding the non zero value. An event of this table is defined as

Event: a combination of a magnitude (index of quantized value) and a run (number of zero values preceding the current non zero value)

In the practical coder, bits are assigned to each event in this table. The following work, however, concentrates on obtaining the entropy of each event and calculating the number of bits per pixel needed to transmit the frame sequence. In addition to the simplest scanning method, some effective techniques to improve entropy value are proposed introducing several ad hoc ideas.

\subsubsection{Simple Scanning}

The most natural and easy way is by scanning all 64 transform coefficients according to the predefined scanning pattern and then generate the events. After the last non zero value has been scanned, an End Of Block (EOB) signal is attached. The zeros which occur after this EOB are discarded in the encoding process. In this algorithm, no information is lost due to the scanning. Ideally, the decoder can reconstruct the block as it is. 


\subsubsection{Zonal Scanning}

Zonal means to scan only a certain number of values out of the transform block and setting all the values which are not scanned to zero. This is a brutal way of limiting the bitrate because some frequency bands will be discarded. For the sake of bitrate reduction, details of the frame sequence are eliminated and only low frequency components will be reconstructed. Two versions of zonal scanning are considered; one scanning only four transform coefficients of the lowest resolution layer, and a second version using sixteen values of the lowest and the middle resolution layer.

\subsubsection{Simple Conditional Scanning}

Looking at Figure 6 , it is pointed out that the likelihood of finding a non zero value in the next higher resolution layer given that there is a zero value is pretty low $\left(p \approx 2 \cdot 10^{-3} \ldots 2 \cdot 10^{-2}\right)$. On the other hand, there is a good likelihood of finding a non zero value in the next higher resolution layer when a non zero value is given $(p \approx 0.2 \ldots 0.3)$. Exploiting this fact, the decision rule will be as follows: the coefficient in a given subband is forced to zero if its corresponding coefficient in lower resolution layer is also zero. As there are always four higher resolution coefficients dependent from a lower resolution coefficient, four coefficients at a time will be forced to zero.

Figure 8 shows how this algorithm works. All the values marked NTS (not to scan) will be forced to zero. In this example, the value of $W_{3}^{H L}$ is zero and therefore $W_{2}^{H L}$ and $W_{1}^{H L}$ are not scanned (marked NTS). Some information is lost as a couple of coefficients may be non zero values. In the subband $W_{2}^{H H}$, one coefficient only is non zero and so only the coefficients at the same location in $W_{1}^{H H}$ are scanned. As it turns out these are all zeros and one might also have skipped them.

\subsubsection{Modified Conditional Scanning}

The conditional scanning is modified compared to the previous version. This version is not only going to force the coefficients to zero but also drop them before scanning the coefficients. So, there will be a lot less zero values around and in the event table runlength should be shorter and more concentrated around zero. At the decoder side, the missing coefficient will be only replaced by zero. No side information is needed to code the missing of some coefficients. While the decoder goes on, it will read the lower resolution layer values and find out which higher resolution values are missing. A variation of this algorithm is to apply zonal scanning at the same time. So, the same conditional scanning technique is used but completely drop the high resolution layer. Only sixteen coefficients will be used therefore. 


\section{Subband Based Approach}

\subsection{Scanning Algorithms}

Different from the previous section, algorithms for scanning entire subbands at a time are defined. The main motivation of scanning entire subimages instead of just 64 coefficients in one block is based on the fact that longer runs are achieved and thus less events to be coded occur. Especially in the high frequency subimages, it is expected that very long zero runs reducing the entropy of the signal a good amount.

\subsubsection{Simple Subband Scanning}

In this simplest case, the image is firstly transformed by the wavelet basis and then separated into its subimages. After that, the transform coefficients are scanned horizontally or vertically according to the strongest correlation in the given subband as described in Section 2. While scanning, different event tables which are tuned to each frequency will be provided.

\subsubsection{Subband Zonal Scanning}

The higher frequency bands are dropped out and also the medium frequency band for one test series similar to the case of the block based approach.

\subsection{Removal of Isolated Non-zero Coefficients}

Isolated non-zero coefficients in higher frequency subbands degrade efficiency of run-length coding although they are often occurred by noises. In order to alleviate this problem, a filter which removes these isolated non-zero coefficients is applied to the medium and high frequency subbands. This filter is block-wise with a fixed size. Inside the window, the number of non zero transform coefficients are counted and compared with a predetermined threshold value. If the number of non zero transform coefficients are smaller than the threshold, these values are forced to zero. The window is of size $n \times 1$ and its orientation is determined according to the results described in Section 2 which refer to the strongest correlation in each subband.

Figure 9 shows the structure of a proposed block-wise non-linear filter. The window is slided over the image and the algorithm is applied. In this example, only if a point appears isolated in a window, this point will be discarded. Had we set the threshold $=2$, only the leftmost window in the second row would have remained unchanged after the filtering operation. All other windows would have been put to zero. 


\section{Simulation Results}

\subsection{Block Based Approach}

\subsubsection{Comparison of Scanning Patterns}

The scanning patterns have been tested on the various CIF sequences. Figure 10 shows an average entropy value of runs for their luminance component. The scanning pattern number in this figure corresponds to the definitions in Section 3.1, but pattern 0 corresponds to the zig-zag scanning.

Among five scanning patterns considered, the pattern 3 performs best slightly followed by the pattern 1. Difference between them results from exploiting correlation between subbands or not although it is very small. The fact that the pattern 4 performs worse than the pattern 3 concludes that directional scanning is more effective than the peano scanning. Poor behavior of the pattern 2 suggests that scanning should be done from lower resolution layers to higher ones. The pattern 0 was originally developed for uniform band splitting techniques such as DCT and does not reflect energy distribution of wavelet transform coefficients sufficiently. Therefore, it is not astonishing to find this pattern performing worst for the wavelet transform.

\subsubsection{Comparison of Scanning Algorithms}

In this subsection, comparison results of the simulations with respect to the scanning algorithms are shown. Taking the result in the previous subsection into account, the scanning pattern 3 is used from now on. A frame of CIF image sequences is composed of $352 \times 288$ pixels for a luminance component and of $176 \times 144$ pixels for two color components. Therefore, $0.042 \mathrm{bit} / \mathrm{pel}$ is corresponding to $64 \mathrm{~kb} / \mathrm{s}$ transmission when a frame rate is assumed to be 10 frames/s.

Firstly, simple scanning and zonal scanning are compared as plotted on Figure 11. Using four pixels only, the performance of the zonal scanning is below the simple scanning for high SNR values. Below $0.057 \mathrm{bit} / \mathrm{pel}$, the zonal scanning with four pixels exceeds the simple scanning. Using sixteen pixels in the zonal scanning guarantees a better performance over the entire range of SNR values. Below 0.037 $\mathrm{bit} / \mathrm{pel}$, the four pixel version is taking the lead.

In the simple conditional scanning, the values which do not need to be scanned are not removed but simply replaced by zeros and less events are generated. Except for a point on the graph at entropy 0.06 bit/pixel, the simple conditional scanning is superior to the zonal scanning using sixteen pixels. At 0.037 $\mathrm{bit} / \mathrm{pel}$, the zonal scanning with four pixels again takes the lead. Below $0.025 \mathrm{bit} / \mathrm{pel}$, the performance of the algorithms becomes similar. This is due to the absence of high frequency components which account for the many events in the higher bit rate environment.

At last, let us have a look on the variation of the conditional scanning which only codes sixteen 
coefficients. Figure 12 shows the improvement of this solution versus the modified conditional scanning using 64 coefficients. From 0.05 bit/pel above, the modified conditional scanning with 64 coefficients takes the lead, but in the lower regions using only 16 coefficients is the better thing to do. At the lower end, the two curves join because almost all high resolution pixels have been discarded.

\subsection{Subband Based Approach}

\subsubsection{Comparison of Scanning Algorithms}

Figure 13 shows comparison results between the simple subband scanning and the subband zonal scanning. The graph looks similar to Figure 11 in the block based approach. Keeping only the four lowest subband images performs well at very low entropy values. But this algorithm tends to saturate at higher SNR values and is surpassed by the other approaches.

Dropping only the three highest subband images gives an even better performance. At around the entropy of $0.05 \mathrm{bit} /$ pixel, this algorithm is $1 \mathrm{~dB}$ better than the one using four subbands. Between 0.04 and $0.08 \mathrm{bit} /$ pixel the seven subband zonal scanning outperforms the simple scanning by $0.5 \mathrm{~dB}$. This entropy range is corresponding to our interesting domain for the target bitrate for $64 \mathrm{kbits} / \mathrm{s}$.

\subsubsection{Effect of the Block-wise Non-linear Filter}

The result of using a block-wise non-linear filter is shown in Figure 14. The best combination of window size and threshold with the least effective choice of these parameters and the simple scanning are compared. The best combination is given with window size 7 and threshold 3 . The gain reaches 1 - $2 \mathrm{~dB}$ compared to the simple scanning approach. Even the least effective of all tested combination with window size 20 and threshold 1 gives a gain compared with simple scanning of approximately 0.3 $-1 \mathrm{~dB}$.

In the field of the tested filters, the couples (window size / threshold) $7 / 3,11 / 3,11 / 5$ shows the best performance around the $0.05 \mathrm{bit} / \mathrm{pel}$ domain which corresponds to the $64 \mathrm{kbit} / \mathrm{s}$. If the ratio of these two values tends to unity, the algorithm becomes much of the zonal scanning scheme, usually saturating for higher SNR / entropy values. If the ratio of window size over the threshold tends to zero, the filter performance is greatly reduced and the resulting curve is much alike simple scanning curve. Thus the best result were obtained with a ratio around $0.3-0.5$, with window sizes ranging between 7 and 11. In Table 1, the average gains for the three frame sequences are summarized. In this experiment, bit rates are around $0.06 \mathrm{bit} / \mathrm{pel}$ (roughly the target bitrate of $64 \mathrm{kbits}$ ). 


\subsection{Discussions}

In Figure 15, summarized results from the previously discussed algorithms are plotted on one graph: the block based modified conditional scanning, the subband zonal scanning, and the subband scanning with or without the block-wise non-linear filter. Up to an entropy of $0.08 \mathrm{bit} / \mathrm{pel}$, the subband scanning with the non-linear filter performs best, with more than $1 \mathrm{~dB}$ advantage over the modified conditional scanning. The subband zonal scanning and the modified conditional scanning have a similar performance between 0.03 and $0.08 \mathrm{bit} / \mathrm{pel}$ but then the zonal scanning saturates. These three algorithms perform better than simple scanning for low bit rate environment.

\section{Conclusions}

In this paper, algorithms for scanning wavelet transformed coefficients are presented. Two different approaches have been investigated, block based and subband based approaches. For low and medium bit rates, the subband scanning with the block-wise non-linear filter performs best. The block based approach with the modified conditional scanning can also give good result in the low bit rate domain. As future works, applying these scanning algorithms on the developed video codec with overlapped motion compensation [8] should be considered.

\section{Acknowledgments}

We would like to thank Dr. Takao Nishitani for his helpful support while doing this work. 
[1] M. Ohta, M. Yano and T. Nishitani: "Wavelet picture coding with transform coding approach", IEICE Trans. Fundamentals, Vol. E75-A, No. 7, pp.776-785, July.1992.

[2] M. Ohta and S. Nogaki : "Hybrid picture coding with wavelet transform and overlapped motioncompensated interframe prediction coding", IEEE Trans. Signal Processing, Vol. 41, No. 12, Dec.1993.

[3] S. Mallat: "A theory for multiresolution signal decomposition: the wavelet representation", IEEE Trans. Pattern Analysis and Machine Intell. Vol. PAMI-11, No. 7, pp.674-693, July.1989.

[4] S. Mallat: "Multifrequency channel decomposition of images and wavelet models", IEEE Trans. Acoust., Speech, Signal Processing, Vol. ASSP-37, No. 12, pp.2091-2110, Dec.1989.

[5] I. Daubechies: "Orthogonal bases of compactly supported wavelets", Comm. Pure Appl. Math., pp.909-996, Nov.1988.

[6] M. Antonini, M. Barlaud, P. Mathieu and I. Daubechies: "Image coding using wavelet transform", IEEE Trans. Image Processing, Vol. 1, No. 2, pp.205-220, Apr.1992.

[7] Y. Q. Zhang and Z. Zafar: "Motion-compensated wavelet transform coding for color video compression", IEEE Trans. Circuit 85 Systems for Video Technology, Vol. 2, No. 3, pp.285-298, Sep.1992.

[8] J. Katto, J. Ohki, S. Nogaki and M. Ohta: "A wavelet codec with overlapped motion compensation for very low bit-rate environment", IEEE Trans. Circuit \& Systems for Video Technology, (to be published).

[9] M. Vetterli: "Multidimensional subband coding: some theory and algorithms", Signal Processing, Vol. 6, pp.97-112, Apr.1984.

[10] J. Woods and S. O'Neil: "Subband coding of images", IEEE Trans. Acoust., Speech, Signal Processing, Vol. ASSP-34, No. 5, pp.2091-2110, Dec.1989.

[11] P. J. Burt and E. H. Adelson: "The Laplacian pyramid as a compact image code", IEEE Trans. Commun., Vol. COM-31, No. 4, pp.532-540, Apr.1983.

[12] M.Vetterli and D.L.Gall: "Perfect reconstruction FIR filter banks: some properties and factorizations", IEEE Trans. Acoust., Speech, Signal Processing, Vol. ASSP-37, No. 7, pp.1057-1071, July.1989. 
[13] O. Rioul and M. Vetterli: "Wavelets and signal processing", IEEE Signal Processing Mag., pp.1438, Oct.1991.

[14] M. Temerinac and B. Edler: "LINC: A common theory of transform and subband coding", IEEE Trans. Commun., Vol. COM-41, No. 2, pp.266-274, Feb.1993.

[15] H. Gharavi: "Subband coding of Video Signals", in Subband Image Coding, John W. Woods Ed., Kluwer Academic Publishers, 1991.

[16] CCITT Recommendation H.261: "Codec for audiovisual services and px64 kbit/s", Geneva, 1990.

[17] F. Bellifemine, A. Capellino, A. Chimienti, R. Picco and R. Ponti: "Statistical analysis of the 2DDCT coefficients of the differential signal for images", Signal Processing: Image Communications, Vol. 4, No. 6, pp.477-488, Nov.1992.

[18] W. Chen and W. Pratt: "Scene Adaptive Coder", IEEE Trans. Commun., Vol. COM-32, No. 3, pp.225-232, Mar.1984.

[19] F. Labriki, V. Haese-Coat and J. Ronsin: "Coding approach by wavelet transform analysis before DCT coding", PCS 91, pp.395-398, Sep.1991.

[20] K. Irie and R. Kishimoto: "Adaptive sub-band coder design for HDTV signal transmission", Proc. of ICASSP'90, pp. 2117-2120, Apr.1990. 


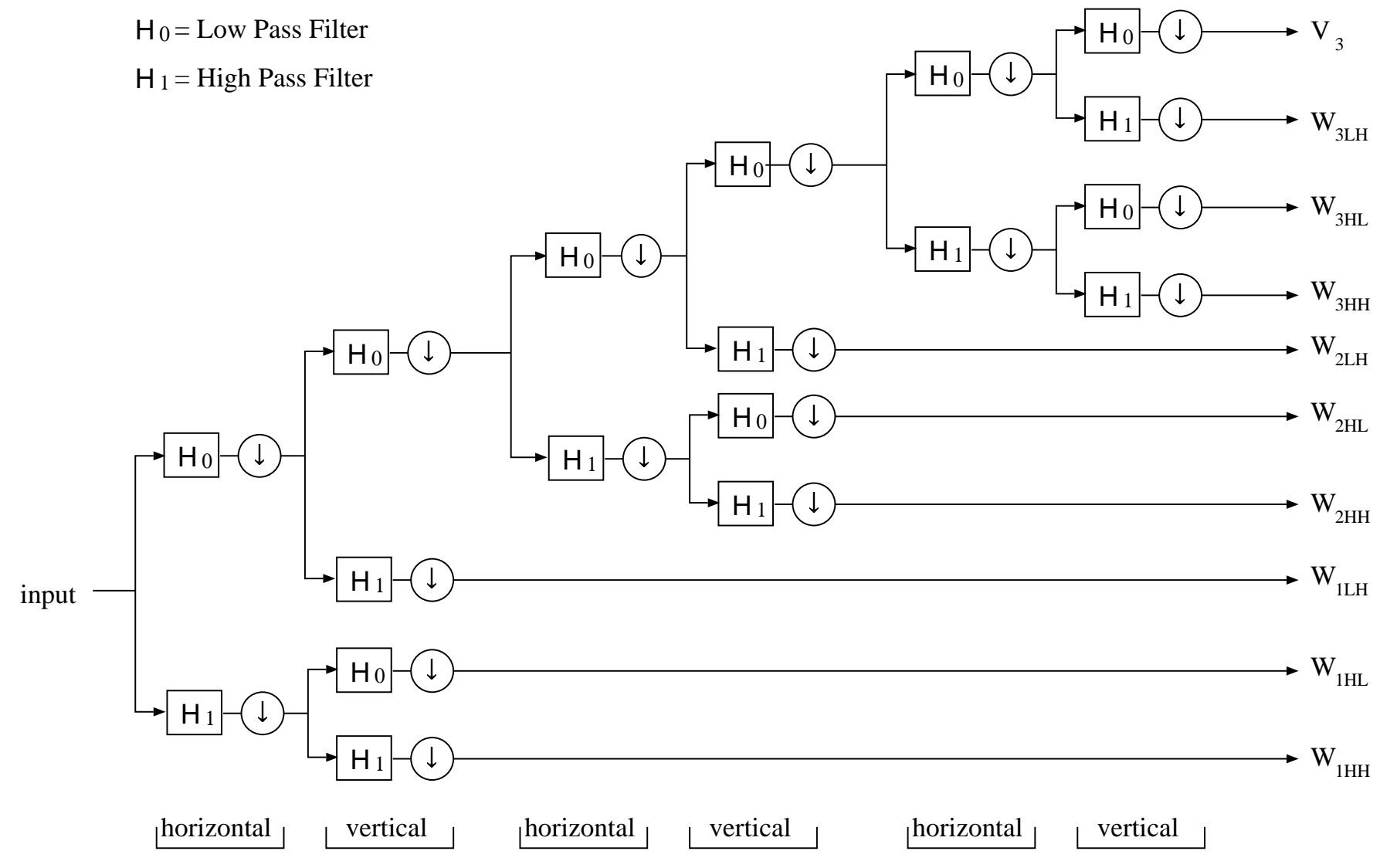

Figure 1: Filterbank which generates the wavelet coefficients 


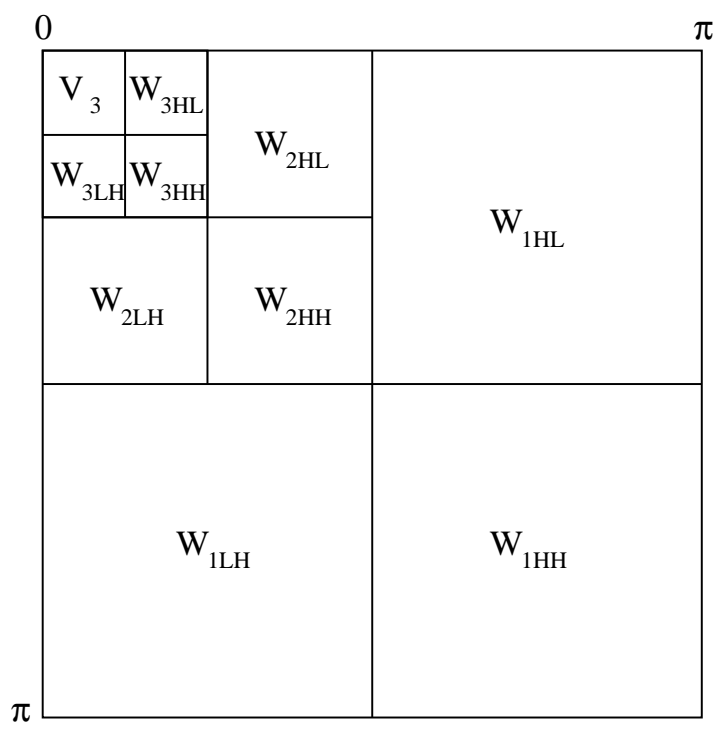

Figure 2: Frequency division of wavelet transform 

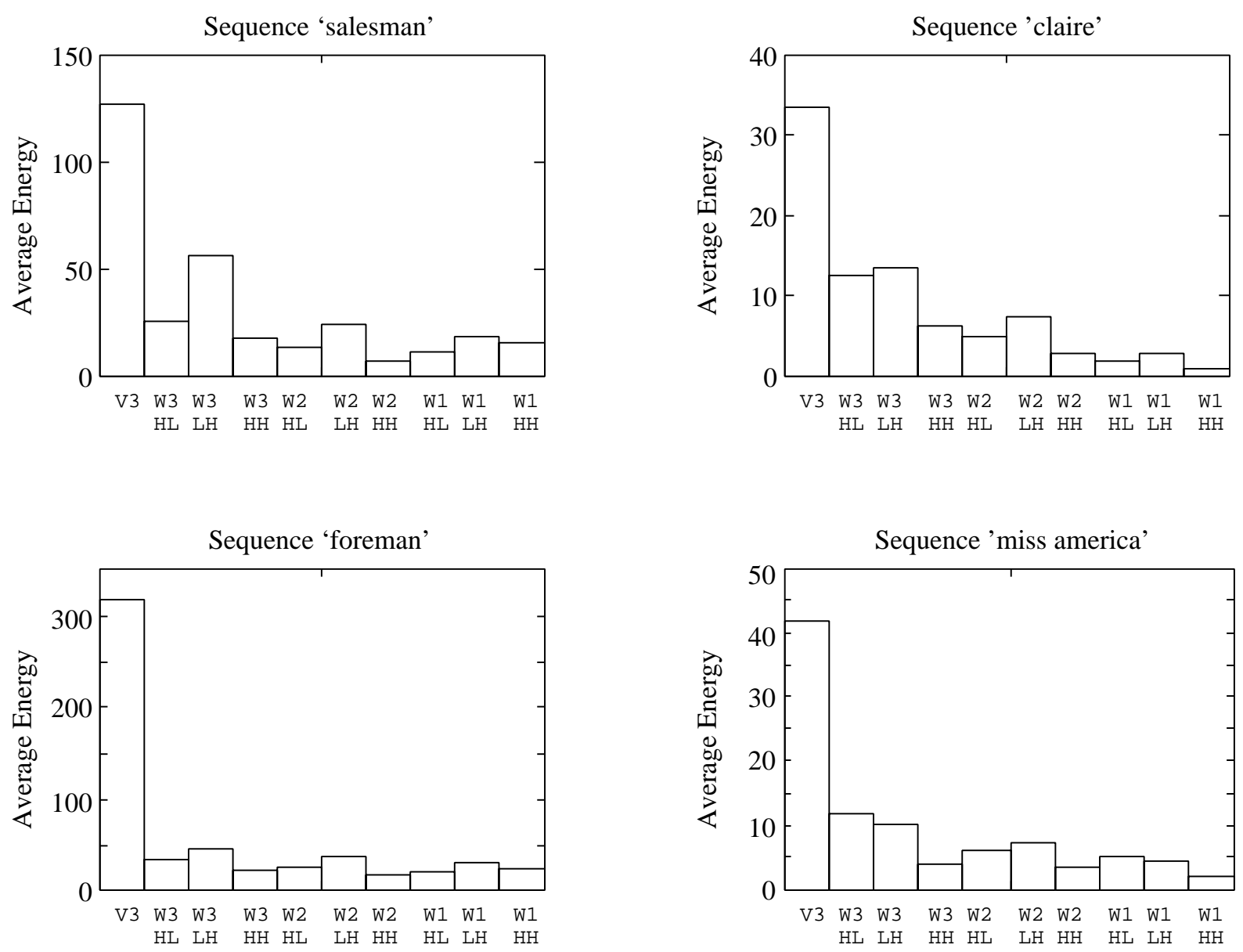

Figure 3: Average energy for CIF image sequences. The energy magnitude differs greatly from input sequence to input sequence, but its order among subbands is almost similar. Most energy is contained in the $V_{3}$. These are calculated for luminance components. 

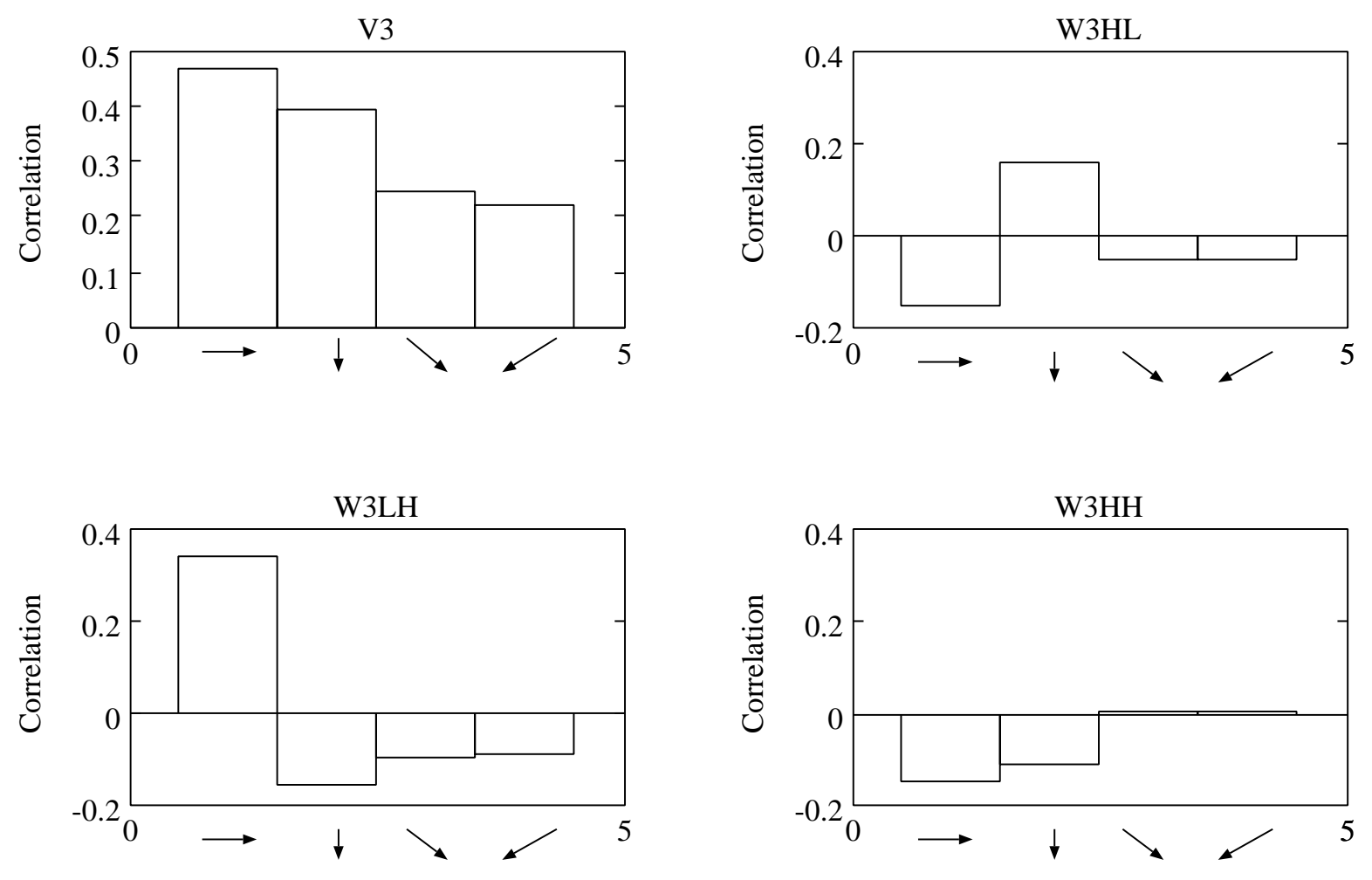

Figure 4: Correlation in four subbands in four directions. The correlation don't change from one resolution layer to another. 


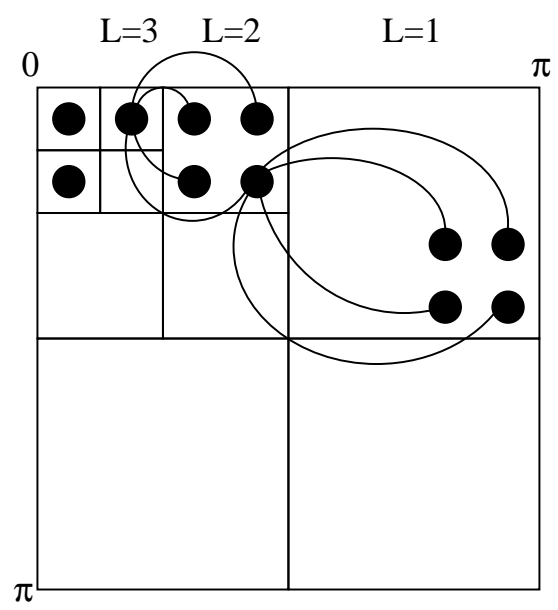

Figure 5: Coefficients between layers are correlated as shown here with the lines. Going from layer 3 to 1 is similar to a zoom-in, seeing more details of the image. 

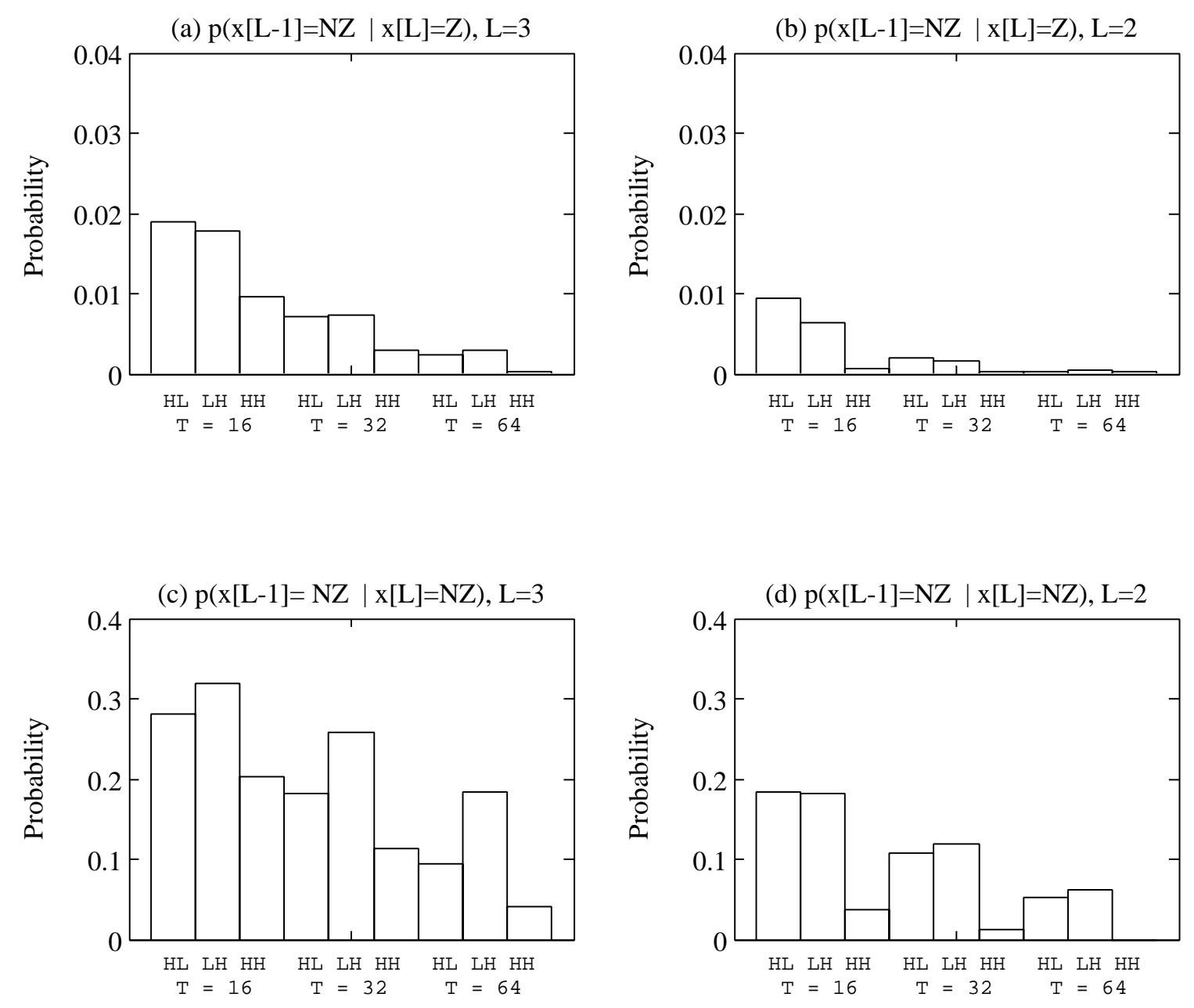

Figure 6: Conditional probabilities between resolution layers. The figure shows the values for various thresholds $T$. 


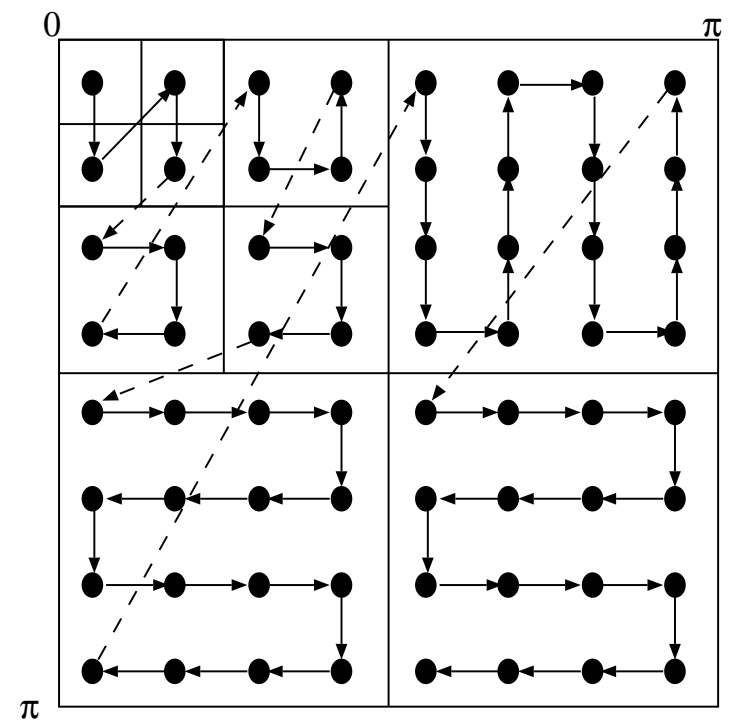

Scanning pattern 1

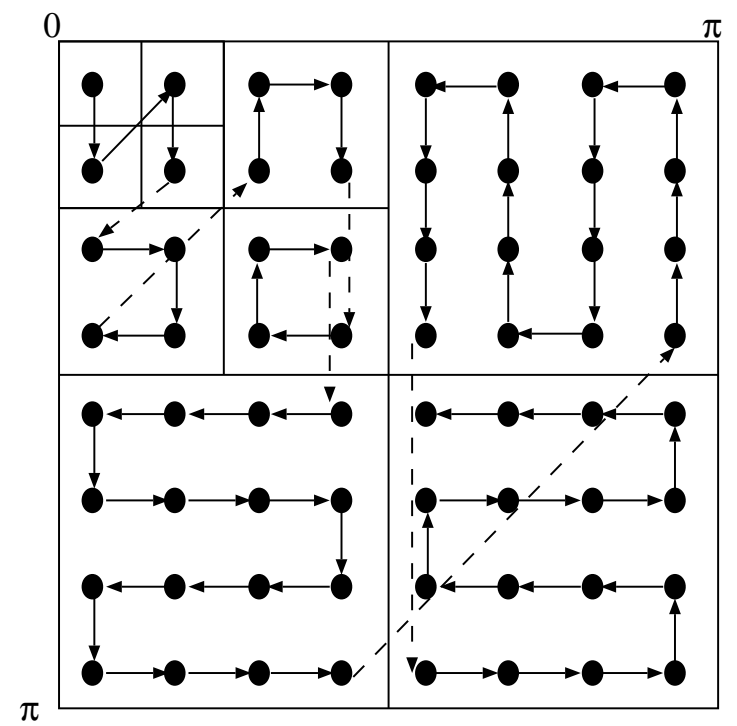

Scanning pattern 3

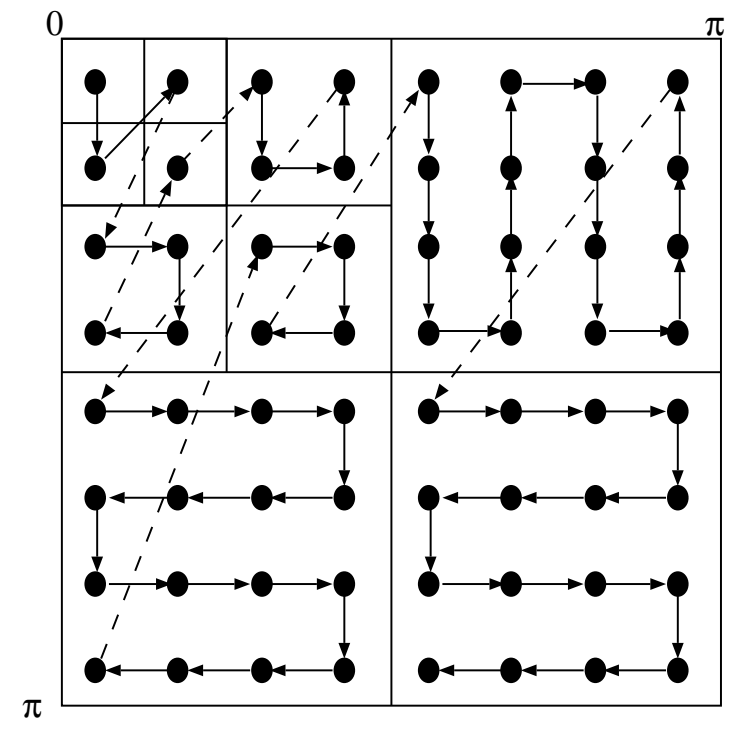

Scanning pattern 2

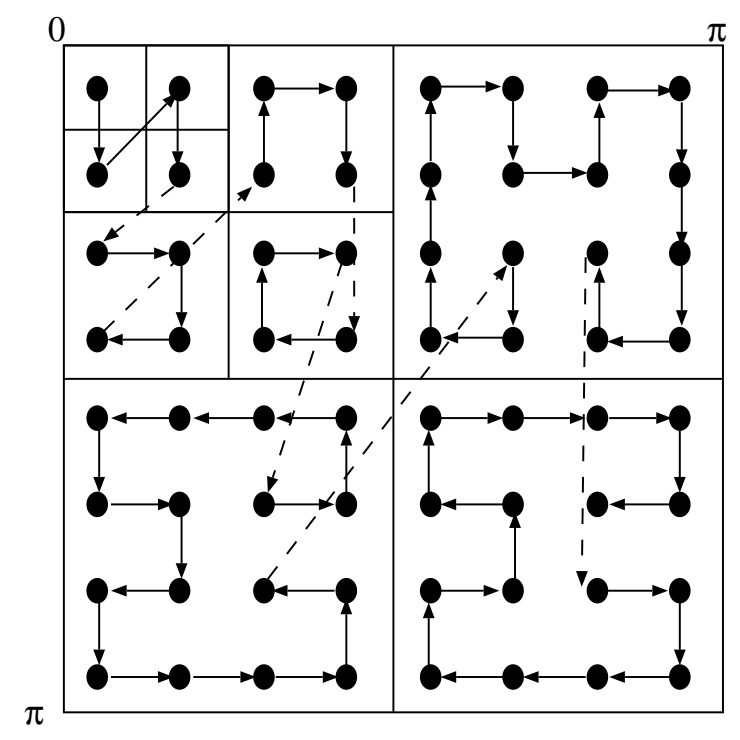

Scanning pattern 4

Figure 7: scanning patterns for block based approach 


\begin{tabular}{|r|r|rr|rrrr|}
\hline 5 & 0 & 2 & 0 & 0 & 0 & 0 & 0 \\
\cline { 1 - 2 }-2 & 1 & 0 & 1 & 0 & 0 & -1 & 0 \\
\cline { 1 - 3 }-4 & 0 & 0 & 0 & 0 & 0 & 0 & 1 \\
-1 & 0 & 0 & 1 & 0 & 0 & 0 & 0 \\
\hline 3 & 1 & 0 & 1 & -1 & 0 & 0 & 0 \\
0 & 0 & 0 & 0 & 0 & 0 & 0 & 0 \\
0 & 1 & 0 & 0 & 0 & 1 & 0 & 0 \\
2 & 0 & 1 & 0 & 0 & 0 & 0 & 0 \\
\hline
\end{tabular}

\begin{tabular}{|c|c|c|c|c|c|c|c|}
\hline 5 & 0 & NTS & NTS & NTS & NTS & NTS & NTS \\
\hline-2 & 1 & NTS & NTS & NTS & NTS & NTS & NTS \\
\hline-4 & 0 & 0 & 0 & NTS & NTS & NTS & NTS \\
\hline-1 & 0 & 0 & 1 & NTS & NTS & NTS & NTS \\
\hline 3 & 1 & NTS & NTS & NTS & NTS & NTS & NTS \\
\hline 0 & 0 & NTS & NTS & NTS & NTS & NTS & NTS \\
\hline 0 & 1 & NTS & NTS & NTS & NTS & 0 & 0 \\
\hline 2 & 0 & NTS & NTS & NTS & NTS & 0 & 0 \\
\hline
\end{tabular}

Figure 8: Example: A block before and after conditional scanning. Values which don't need to be scanned are marked Not To Scan (NTS). 


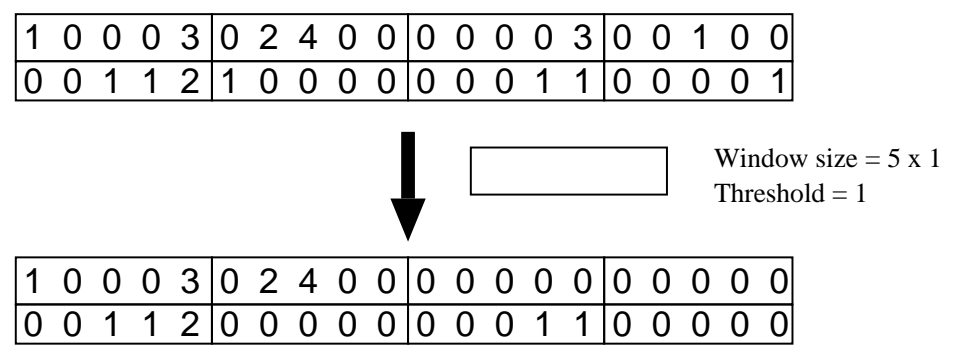

Figure 9: Passing a $5 \times 1$ window over a part of an image with stronger horizontal correlation. Because of the threshold $=1$, only if there is a single point in the window this point will be discarded. The higher the threshold, the more coefficients will be omitted. 


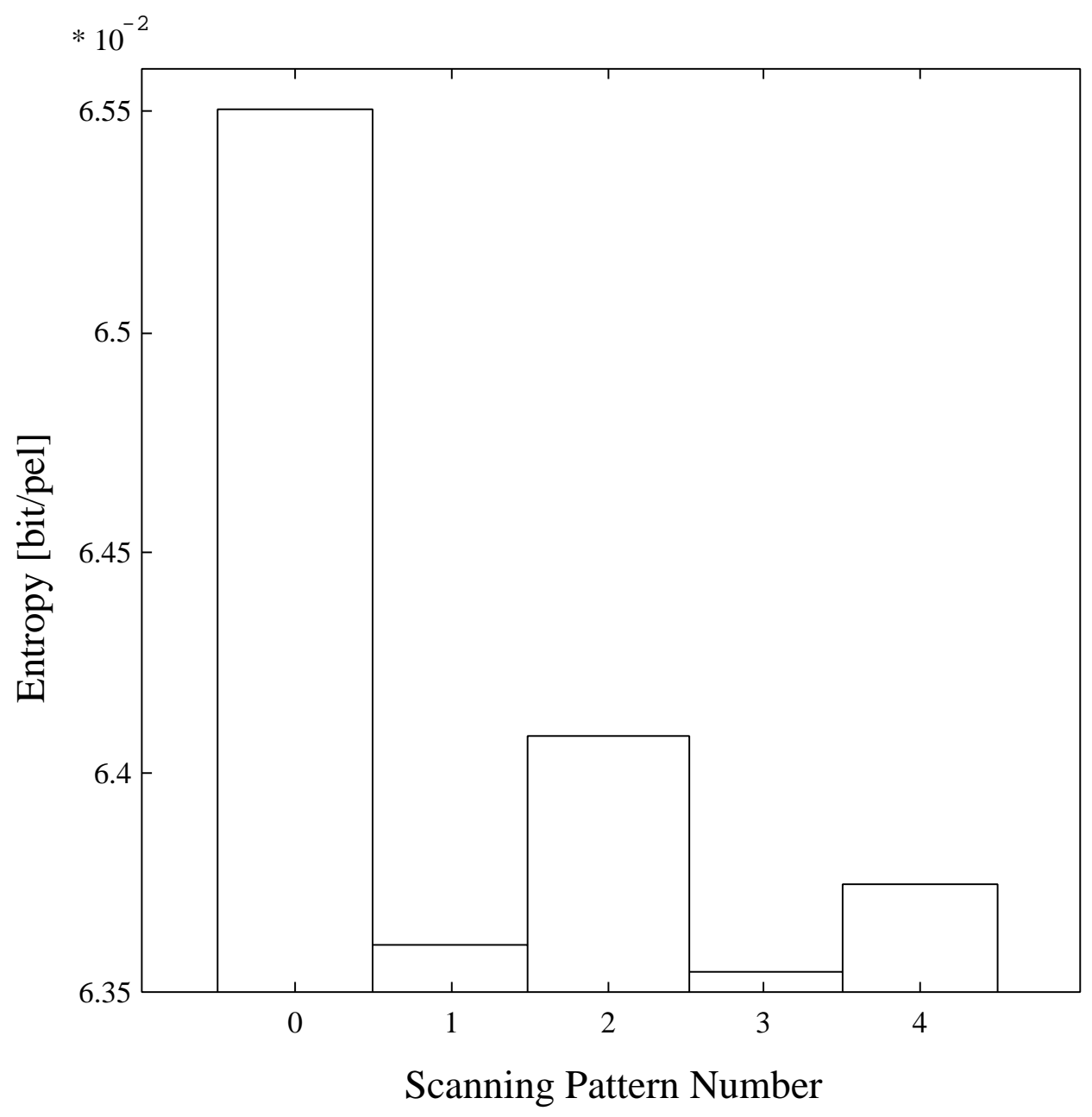

Figure 10: Comparison of the scanning pattern for the luminance component. 


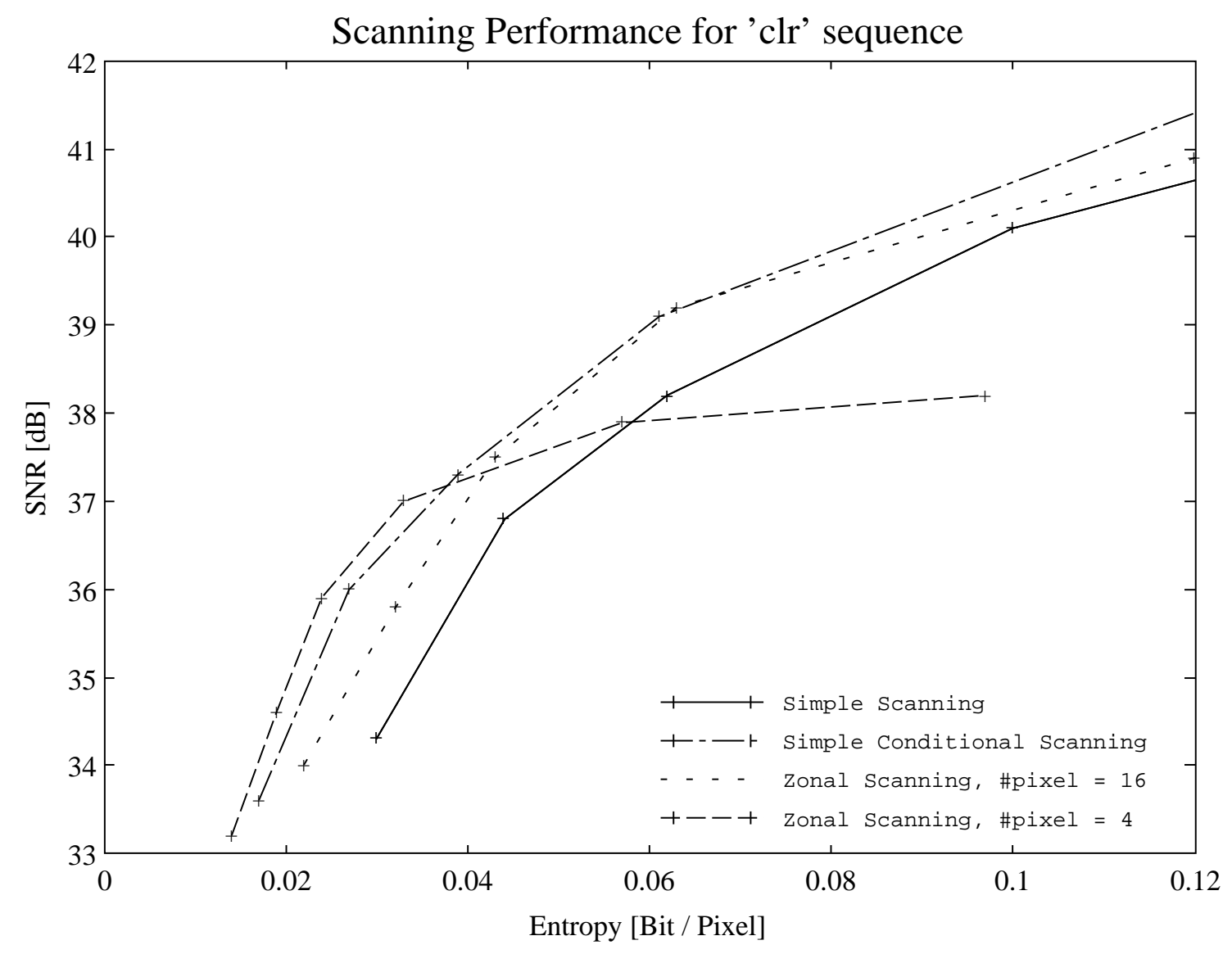

Figure 11: Comparison between zonal scanning and simple conditional scanning 


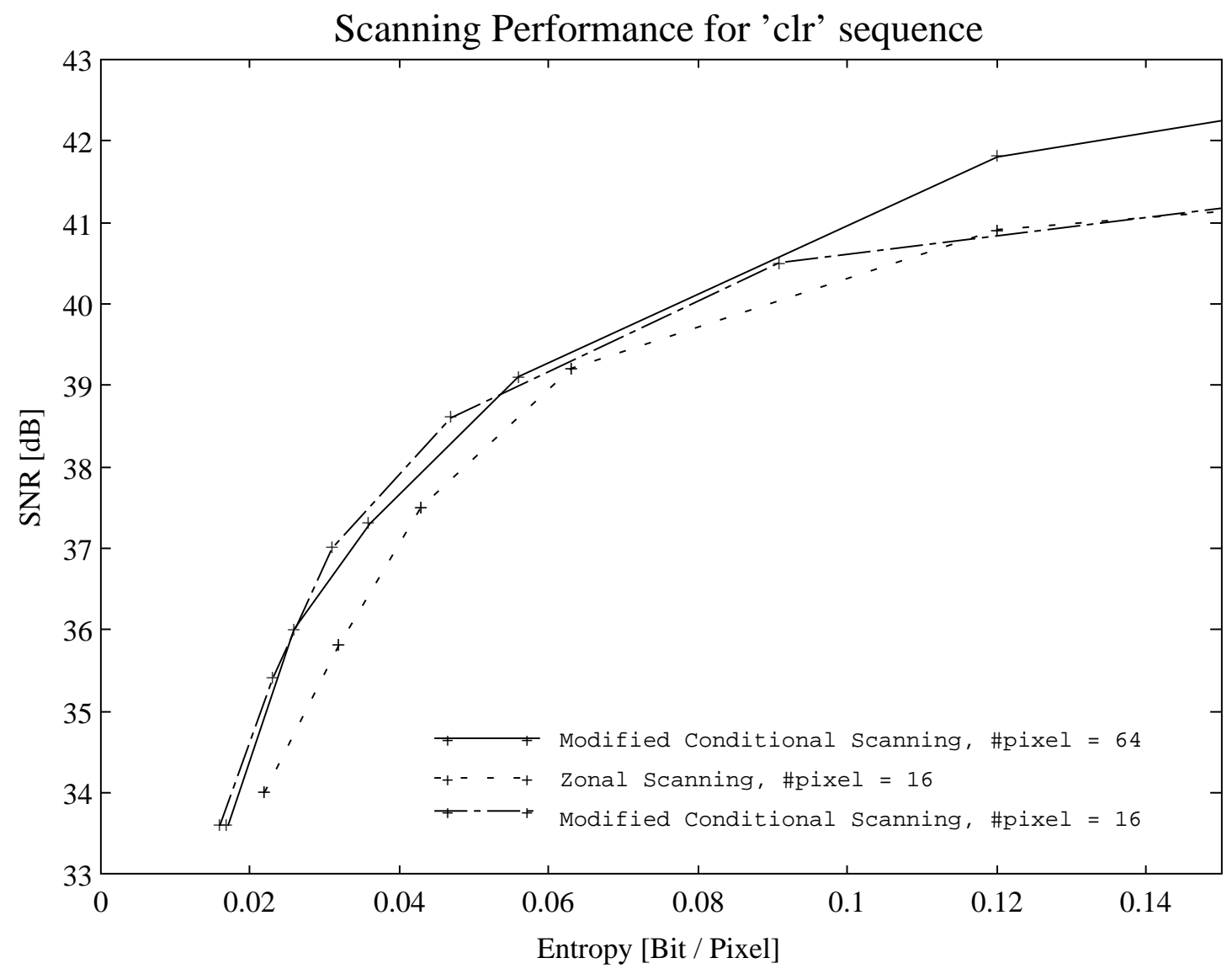

Figure 12: Comparing modified conditional scanning 


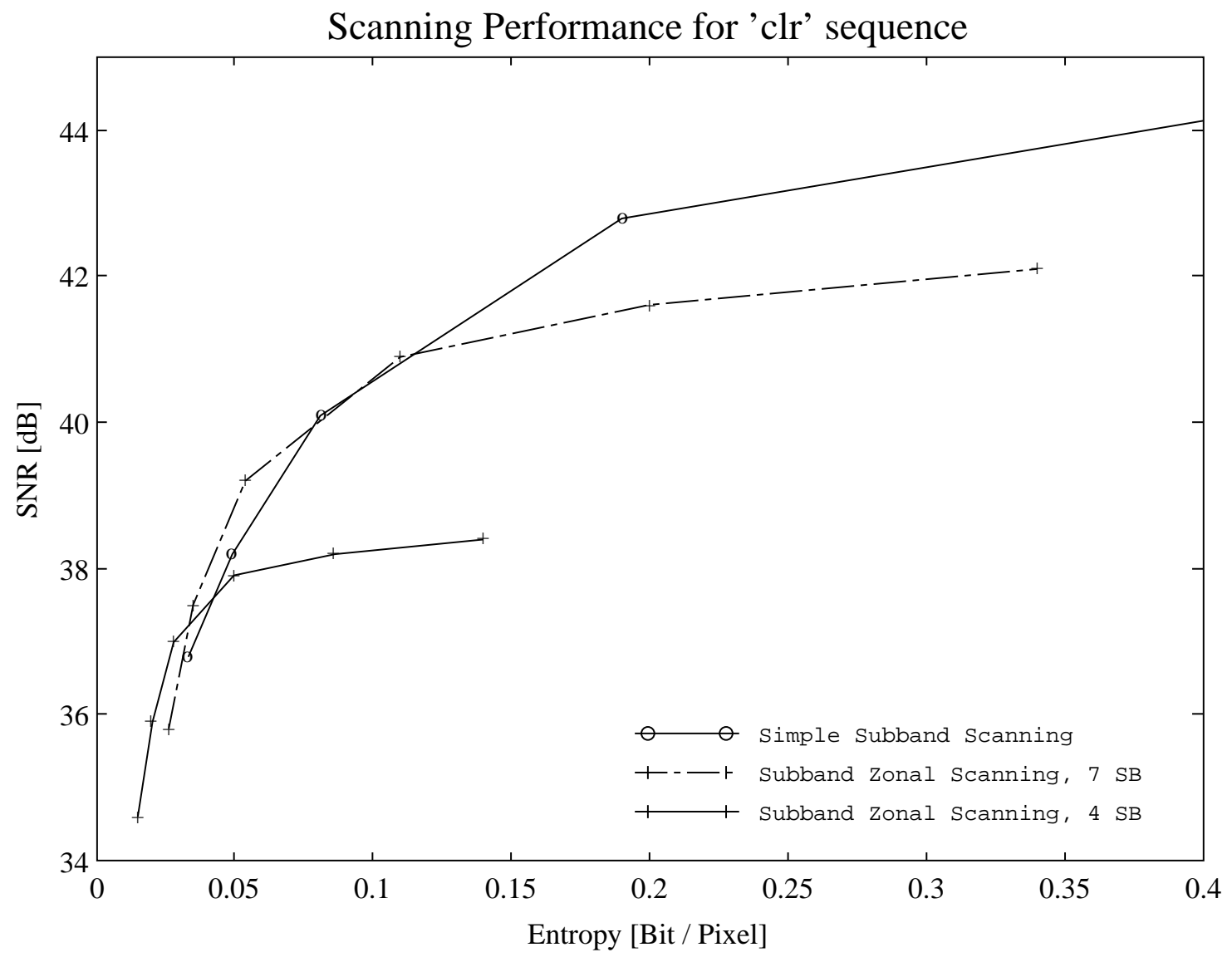

Figure 13: Simple subband scanning and zonal subband scanning. 


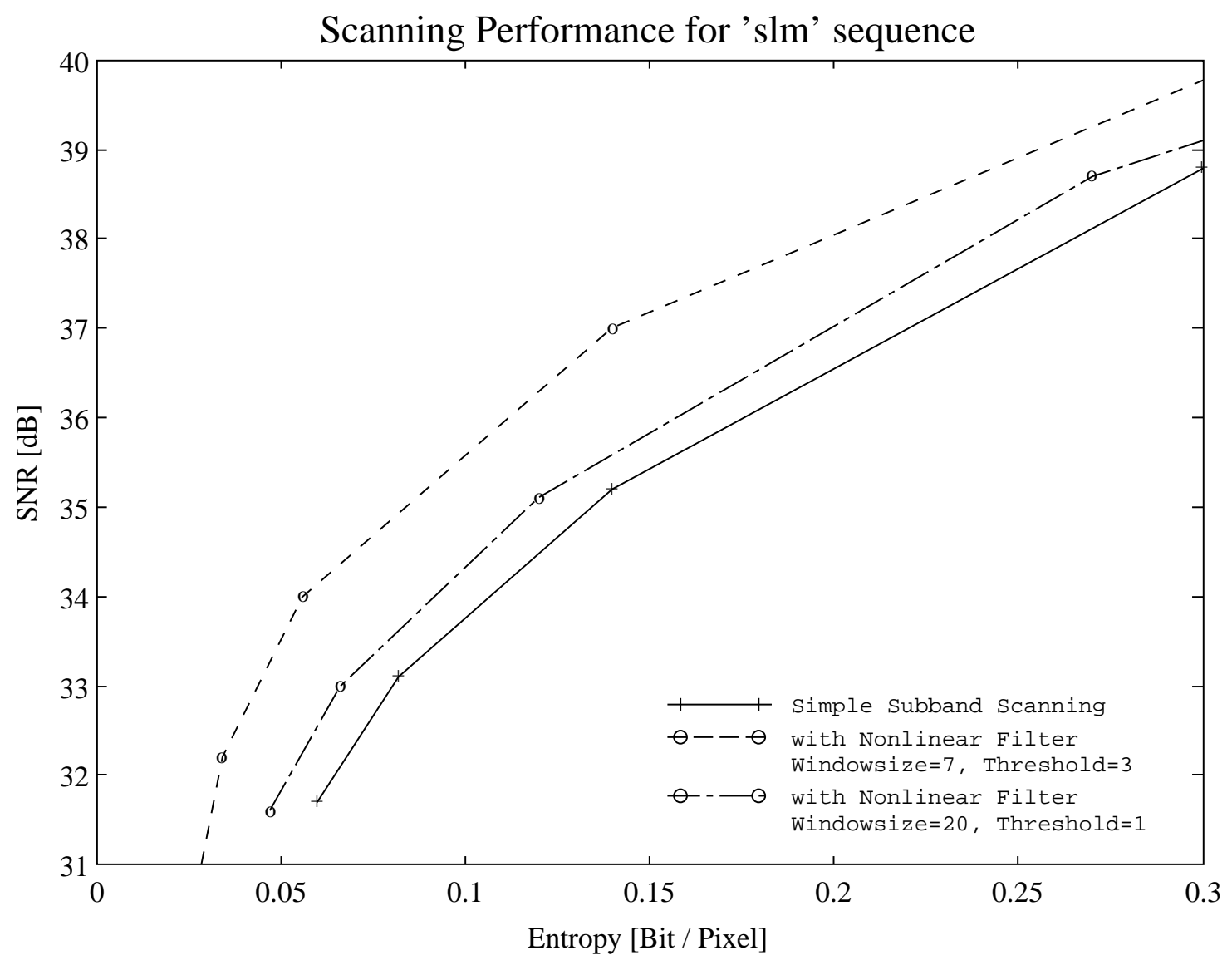

Figure 14: Effect of the block-wise non-linear filters. The best filter has window size 7 and threshold 3 and the improvement is $1-2 \mathrm{~dB}$. 


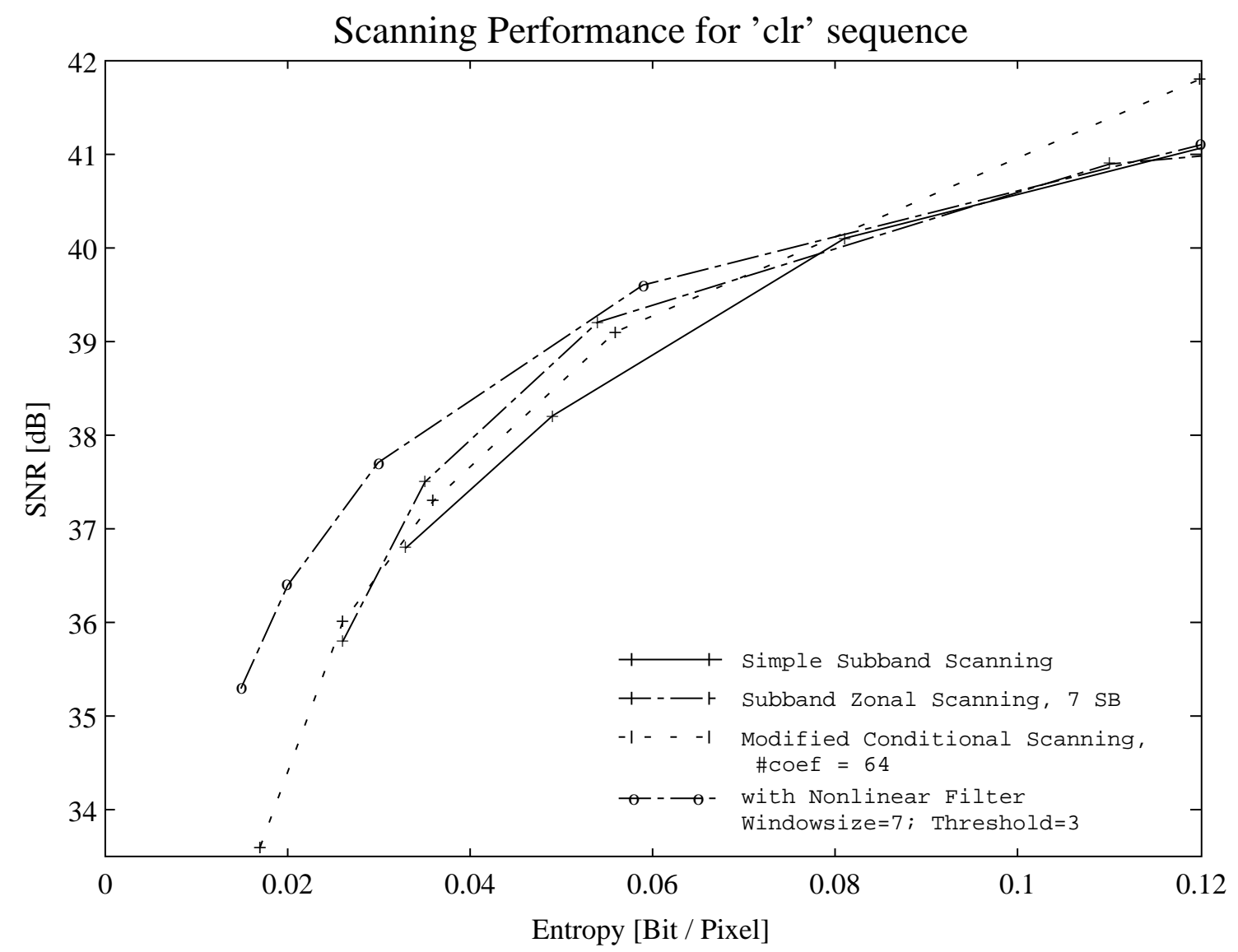

Figure 15: Comparison of the algorithms discussed in this report. In the low bit rate domain, the subband scanning with the non-linear filter performs best. 


\begin{tabular}{|c|c|c|c|c|}
\hline \multicolumn{5}{|c|}{ Average gain [dB] } \\
\hline \hline \multirow{2}{*}{ Threshold } & \multicolumn{4}{|c|}{ Windowsize } \\
\cline { 2 - 5 } & 5 & 7 & 11 & 20 \\
\hline 1 & 1.10 & 1.00 & 0.83 & 0.73 \\
3 & 1.47 & 1.60 & 1.47 & 1.50 \\
5 & - & 1.37 & 1.40 & 1.47 \\
\hline
\end{tabular}

Table 1: Average gains for the non-linear block-wise filter compared with the simple scanning. The combination of window size 7 with threshold 3 gives the best result. The combination $5 / 5$ is in fact the zonal scanning as all coefficients will be discarded. 